\title{
A complicated path to the CRMO diagnosis - case of a 9 year old girl whose story comes full circle
}

\author{
Joanna Świdrowska-Jaros ${ }^{*}$ (D) and Elżbieta Smolewska
}

\begin{abstract}
Background: Chronic recurrent multifocal osteomyelitis (CRMO) is a rare idiopathic autoinflammatory bone disease that mostly affects children and adolescents. It is a diagnosis of exclusions since no clinical signs and symptoms are pathognomonic. Radiological tests are often essential, but bone biopsy may be needed in unclear cases.

Case presentation: A 9-year-old Caucasian girl with a history of bone pain. The data from the history and results of laboratory tests suggested osteomyelitis, but no adequate response to the treatment was observed. A number of imaging tests did not confirm the diagnosis, therefore a bone biopsy was necessary.

Conclusions: Differential diagnosis of CRMO is challenging and it is based on exclusions. Since it might be misdiagnosed or mistreated, bone biopsy should be considered in patients reporting bone pain who are unresponsive to treatment.
\end{abstract}

\section{Background}

Chronic recurrent multifocal osteomyelitis (CRMO) is an autoinflammatory bone disease of unknown origin, that mostly affects children and adolescents [1]. Symptoms of CRMO might be varied - from asymptomatic single-bone involvement to chronic, recurrent, multifocal inflammation with systemic symptoms such as weakness, febrile states and weight loss [2]. Bone lesions are located in various skeletal sites, mainly in long bones metaphyses (tibia, thigh, arrow), pelvic bones, spine, clavicle or mandible [3]. A diagnostic path is complicated and includes laboratory tests and variation of imaging examinations. Routine inflammation markers are often in a normal range and the imagining may result unclear - in that cases, bone biopsy might be needed. Differential diagnosis is difficult and contains malignancies, chronic infections or other systemic diseases $[4,5]$.

\section{Case presentation}

A 9-year-old Caucasian girl was referred to the Department of Paediatric Cardiology and Rheumatology of Medical University of Lodz with a history of bone pain

* Correspondence: j.swidrowska@gazeta.pl

Department of Paediatric Cardiology and Rheumatology, Medical University of Lodz, Łódz, Poland

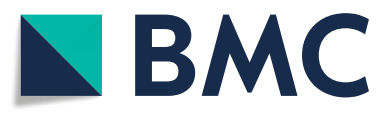

for the last one month. The pain was localized in the lower extremities, waking up at night and making it difficult to walk. Any peripheral joint oedema had been observed. The patient was otherwise healthy, no fevers, chills or weight loss were reported. She had no family history of bone or joint abnormalities, including tumors. She had no history of trauma or any triggering factor for the onset of the pain.

At the time of initial evaluation, she was reporting pain throughout the day and night. Pain was quantified as 8 out of 10 in the visual analogue scale (VAS). The left ankle was slightly swollen and was tender to touch, but no increase of the local temperature was observed. The active and passive motion ranges of the lower extremity joints - such as hips, knees and ankles - were decreased, due to severe pain. In otherwise general physical exam including skin and neurological exam was normal, no infection foci were found elsewhere in the body.

Her initial blood work revealed high rates of inflammation markers (CRP 119,1 mg/l $N<5,0$; ESR $135 \mathrm{~mm} /$ h) with normal hematological and biochemical parameters (including lactate dehydrogenase, alkaline phosphatase or uric acid). At the day of admission, ultrasound examination of peripheral joints of the lower limbs was performed, no joint effusion and any other joint

(c) The Author(s). 2019 Open Access This article is distributed under the terms of the Creative Commons Attribution 4.0 International License (http://creativecommons.org/licenses/by/4.0/), which permits unrestricted use, distribution, and reproduction in any medium, provided you give appropriate credit to the original author(s) and the source, provide a link to the Creative Commons license, and indicate if changes were made. The Creative Commons Public Domain Dedication waiver (http://creativecommons.org/publicdomain/zero/1.0/) applies to the data made available in this article, unless otherwise stated. 
inflammation features were found. Empirical antibiotic (ceftriaxone) therapy together with non-steroidal anti-inflammatory drugs was implemented, however, the inflammatory markers' levels were worsening in the control laboratory tests. Antibiotic treatment was extended with aminoglycoside, the girl reported aggravating of the night pain, migrating from bone to bone, night sweats were additionally observed. Blood culture was negative, but serologic tests had detected positive antibody titers to various pathogens (EBV, Yersinia spp., Mycoplasma and Chlamydia pneumoniae, Salmonella spp., Borrelia burgdorferi) - that was probably associated with immunological disorders.

It was decided to perform isotope bone scan (Fig. 1) revealing increased uptake in the femur, humerus and tibia, that could correspond to bone inflammation. Despite prolonged antibiotic treatment, no therapeutic effect was achieved - inflammatory parameters were increasing, gradual anemization appeared. Ultrasound examination of peripheral joints was made again (Figs. 2, 3), showing a large progression of lesions. Destruction of distal metaphysis of the left tibia and the outline of the left femoral head was found with the inflammation features penetrating throughout the bone. A suspicion of proliferation process was made based on the radiograph (Fig. 4) and a computed tomography of the ankle area (Figs. 5, 6). It

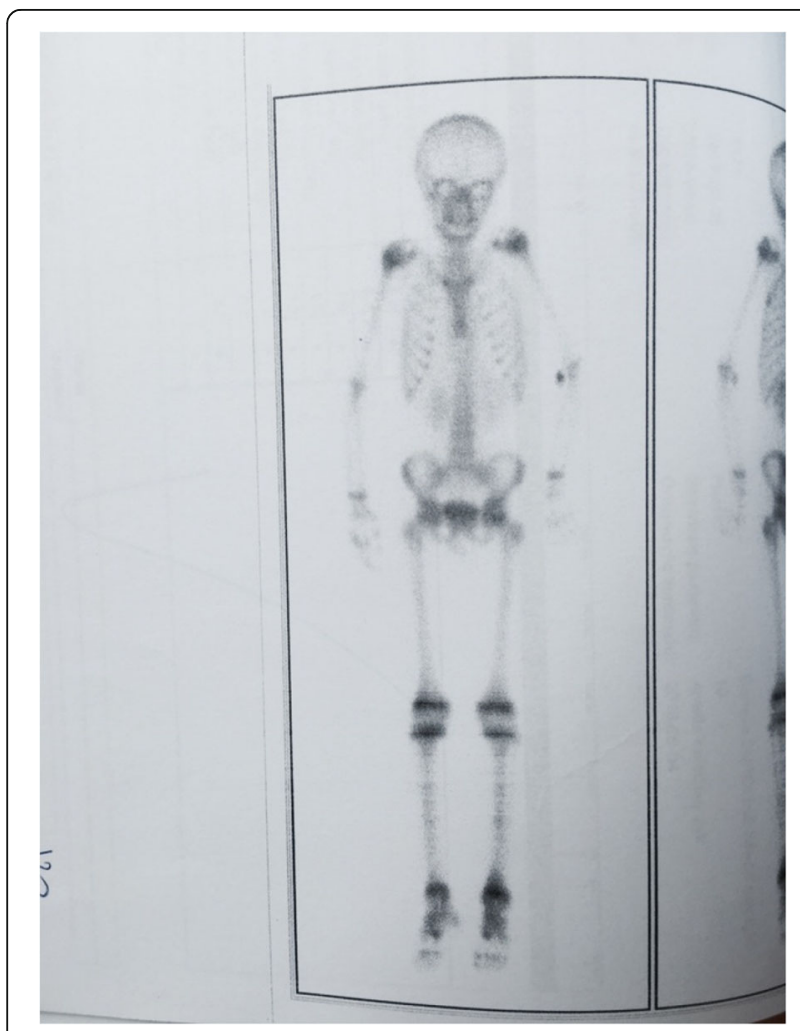

Fig. 1 Isotope bone scan revealing increased uptake in the femur, humerus and tibia was decided to perform the bone marrow biopsy, which showed no significant abnormalities. No non-specific tumor markers (NSE, AFP, beta-HCG and chromogranin A) were found positive. NMR-DWIBS examination (Figs. 7, 8 and 9) revealed numerous osteolytic foci suggesting a disseminated tumor process - with no apparent pathological mass in both the abdominal cavity and the chest. The decision was made to transfer the patient to the Department of Pediatric Oncology and Oncologic Surgery of Institute of Mother and Child in Warsaw, where the antibiotic treatment had been suspended and bone biopsy was performed. Since the malignancy was ruled out and no bacterial culture were positive, the diagnosis of CRMO was considered at this point. The girl was referred back to our Department, where the antibiotic treatment was stopped as ineffective, and nonsteroidal anti-inflammatory medication (naproxen) was again introduced. Due to the unsatisfactory control of the inflammatory process, systemic steroids and sodium pamidronate infusions were used, resulting in significant clinical improvement, relief of pain and decrease in the value of inflammation markers. NMR-DWIBS was performed after two cycles of treatment with bisphosphonates and revealed a large regression of osteolytic lesions including the major focus in the metaphysis of left tibia. No new osteolysis foci were observed.

\section{Discussion}

Diagnostic path of CRMO is complicated due to the overlap of clinical and imaging findings [6]. It often remains a diagnosis of exclusions between tumors and infectious arthritis [7]. Unfortunately, there are various criteria according to different authors. The latest criteria belongs to Roderick et al (Bristol diagnostic criteria for CRMO). Authors suggest, that CRMO might be suspected in case of a bone pain with or without swelling and without significant features of infection, with the typical radiological findings (lytic areas, sclerosis, new bone formation) and - if the disease is multifocal, with no CRP level elevation, but - if the disease affects one bone - with CRP level greater than $30 \mathrm{~g} / \mathrm{l}$ and the bone biopsy showing inflammatory changes with no bacterial growth while not on antibiotic therapy [8]. Manson et al proposed the criteria that includes remission and exacerbation of signs and symptoms for at least 6 months, lack of an identifiable cause, lack of response to antibiotics for at least one month and the chronic, nonspecific inflammation consisting of lymphocytes, plasma cells and histiocytes at histopathologic examination [9]. Otherwise, Beretta-Piccoli et al advise that CRMO can be diagnosed if the disease course lasts at least 3 months in duration, there is a bioptical evidence of chronic bone inflammation with the exclusion of other diseases, and there is a failure to cultivate an organism [10]. 


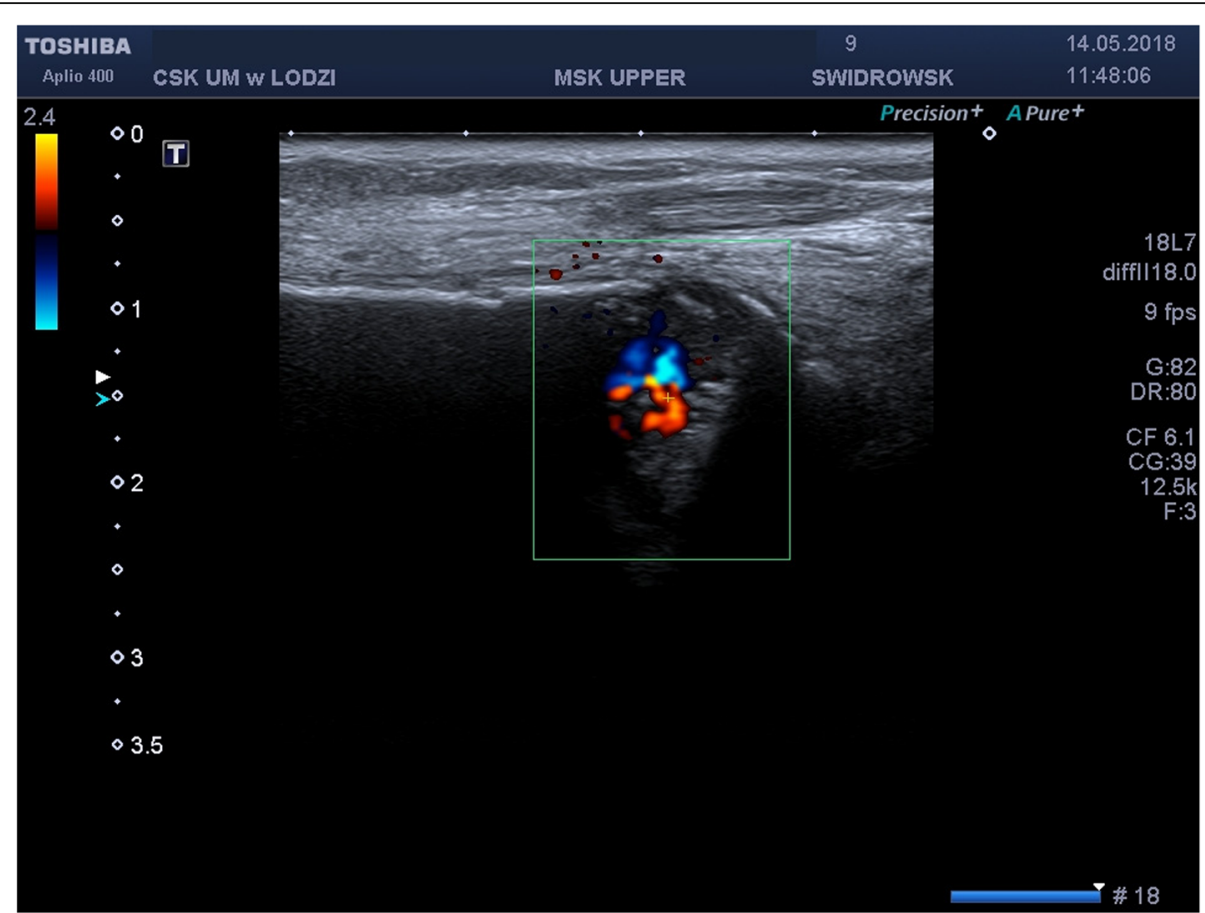

Fig. 2 Ultrasound examination of an ankle area showing destruction of distal metaphysis of the left tibia

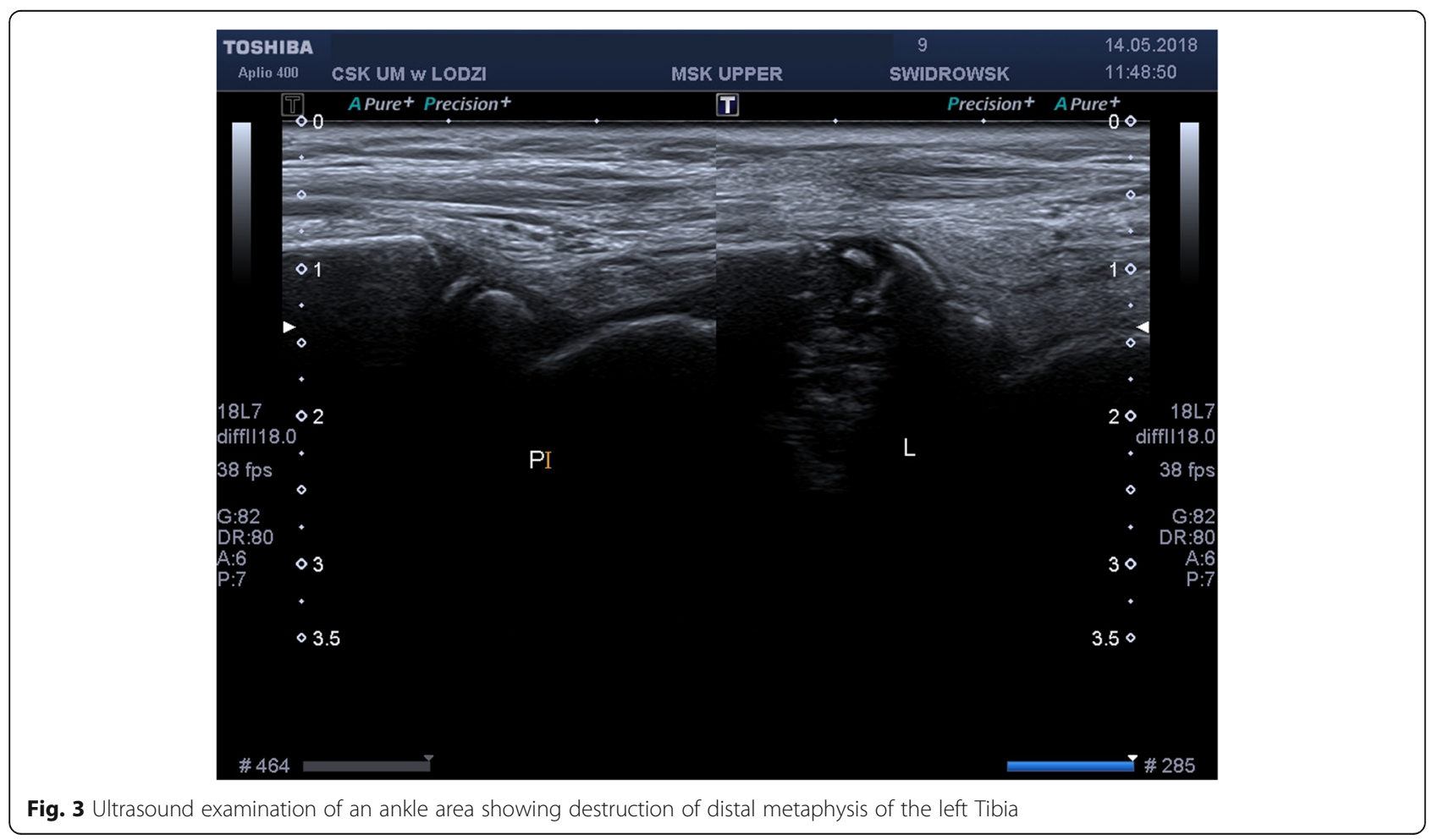




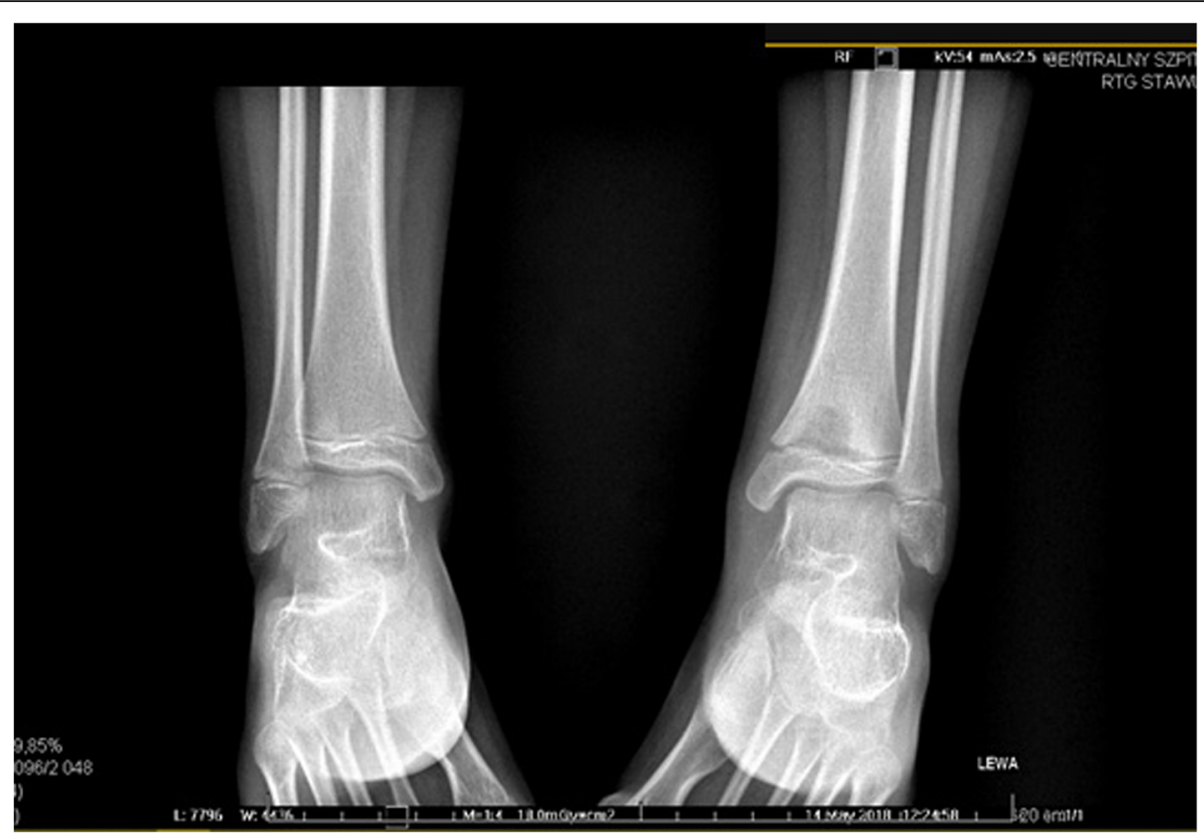

Fig. 4 Radiograph showing a large lytic lesion in the metaphysis of left tibia

Exclusion of other etiologies are the main purpose on the diagnosis of CRMO, since no specific diagnostic biomarkers are available [11]. Malignancies are the first to rule out by the clinician - Ewing's sarcoma, osteosarcoma, Langerhans cell histiocytosis [12-15]. Multifocal involvement is helpful in establishing the diagnosis, however - when there is a single or atypical lesion, the diagnostic pathway might be challenging.

Routine inflammation markers are in a normal range in the majority of affected subjects. However, Brown et al and Catalano-pons et al reported inflammatory

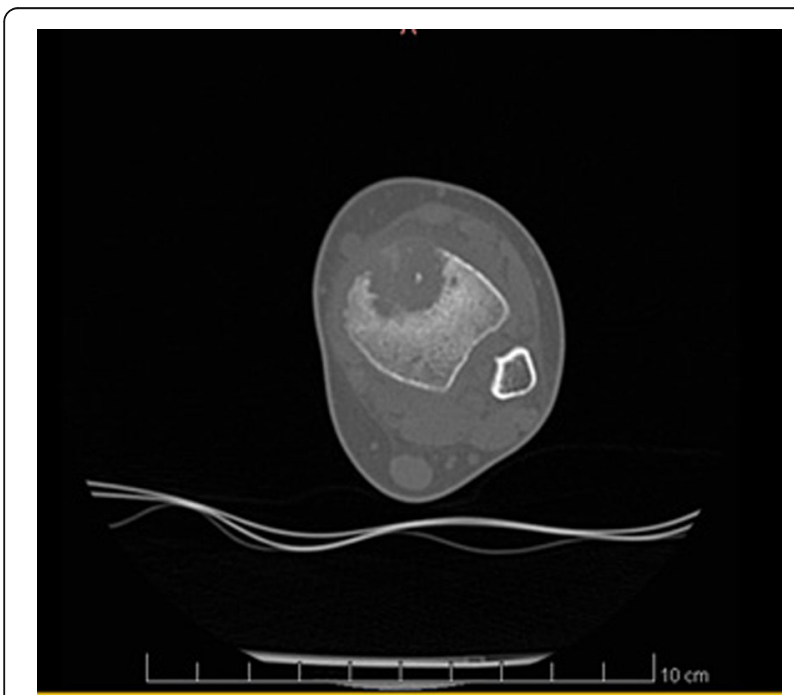

Fig. 5 CT scan showing osteolytic lesion of the metaphysis of left tibia markers increased in more than a half of the examined patients [16]. In our case - highly elevated CRP and ESR got the clinician confused and suggested infectious osteomyelitis. Since there was no response to antibiotic treatment, further investigation was performed. Imaging in CRMO is not clear though. Having a multifocal localization - radioisotope bone scan may be a useful tool in establishing the diagnosis and identifying clinically silent lesions that are may be present at the initial stage $[17,18]$. Radiographic evaluation can be

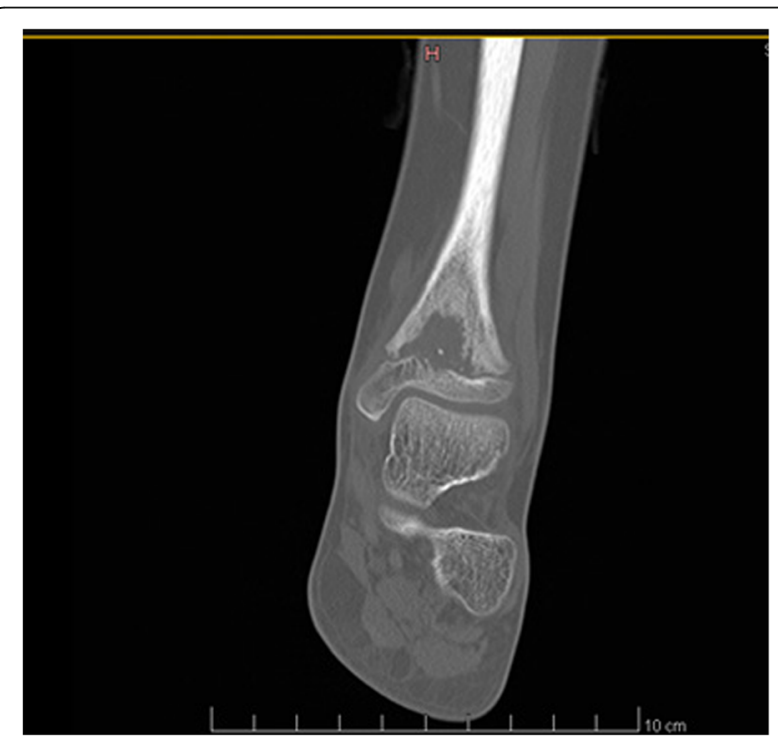

Fig. 6 CT scan showing osteolytic lesion of the metaphysis of left tibia 


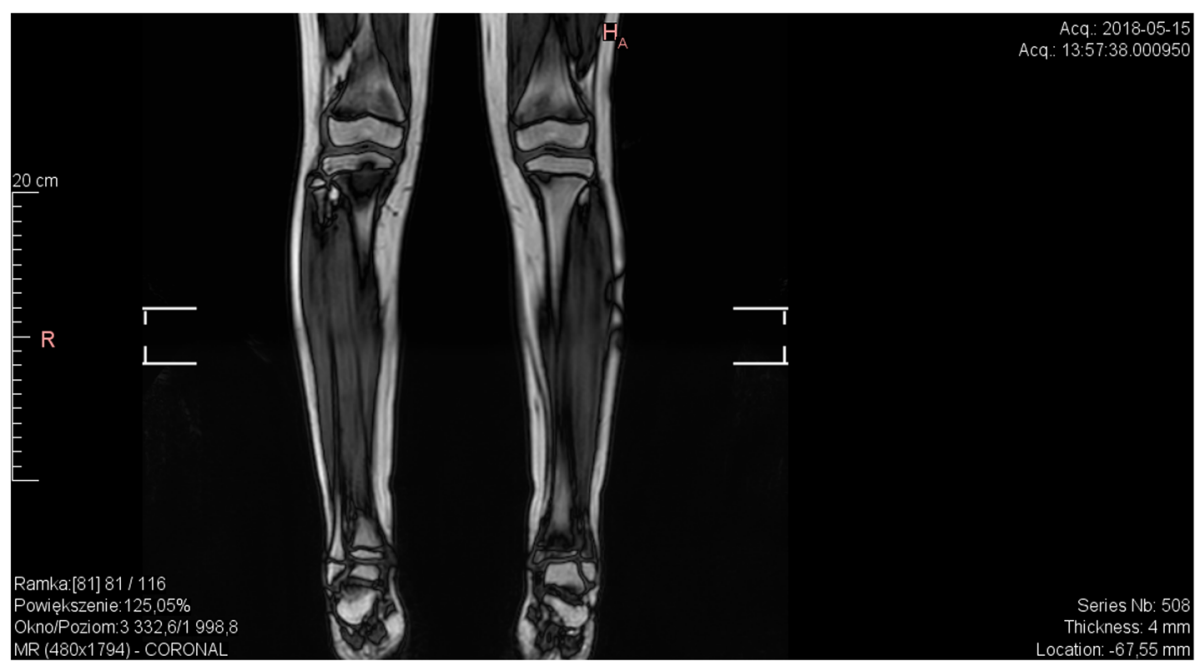

Fig. 7 Whole body NMR revealing multiple foci of elevated marrow signal

characteristic but not pathognomonic, although computed tomography has a limited role in the diagnosis of CRMO [7, 15]. Both methods work parallel and might demonstrate decalcification or osteolysis, periosteal reaction and the bone destruction. Initial radiographs show metaphyseal disease (lytic lesions adjacent to the growth plate). MRI can be useful to clearly characterize the type of lesions and may help in determining the best biopsy location [19]. Moreover, the whole body MRI could assist in evaluating and excluding a pathological mass wherever in the body [20, 21]. The proper image strategy, suggested by Handrick et al, should be as follows: radiographs, bone scintigraphy, MR imaging [22]. In case of our patient, all of the imaging methods mentioned above were used, but no diagnosis was set. To rule out both - chronic bacterial osteomyelitis and malignancy - definite diagnosis relies on histopathological confirmation done by bone biopsy.

Our patient fulfills most of the clinical features that are present in other previous reports. CRMO occurs mainly in children and adolescents and affects the girls aged to be about 10 . The most often affected location

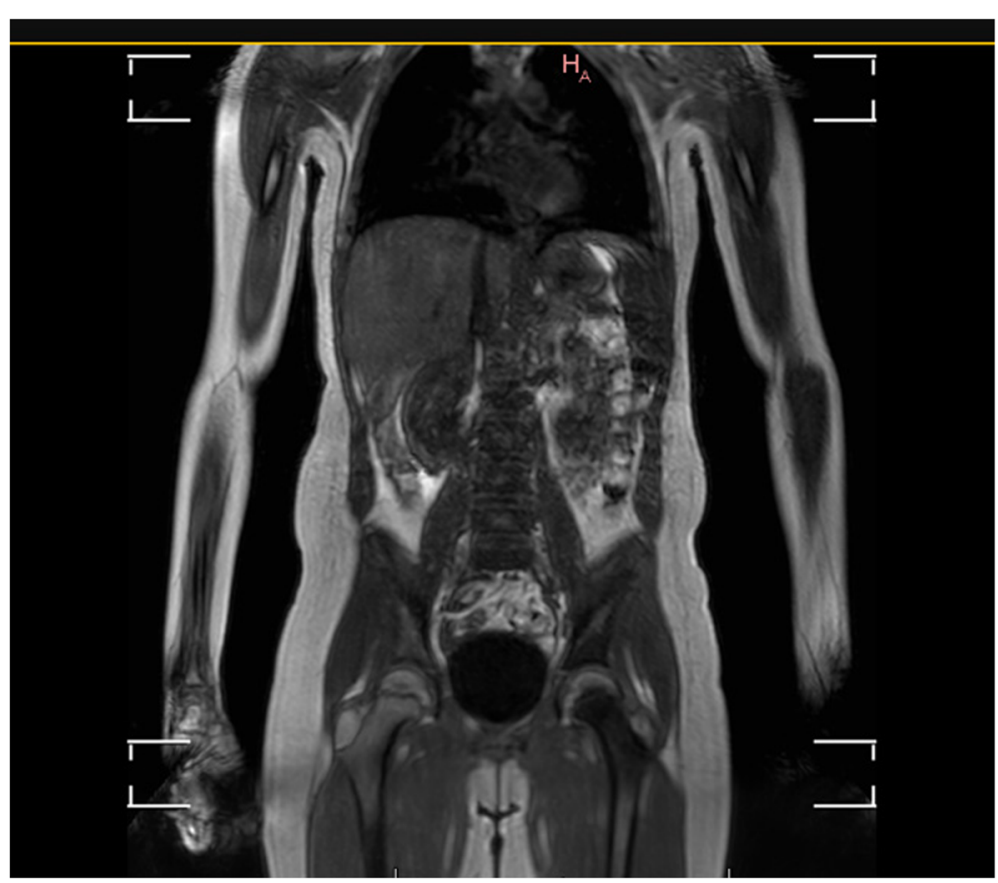

Fig. 8 Whole body NMR revealing multiple foci of elevated marrow signal 

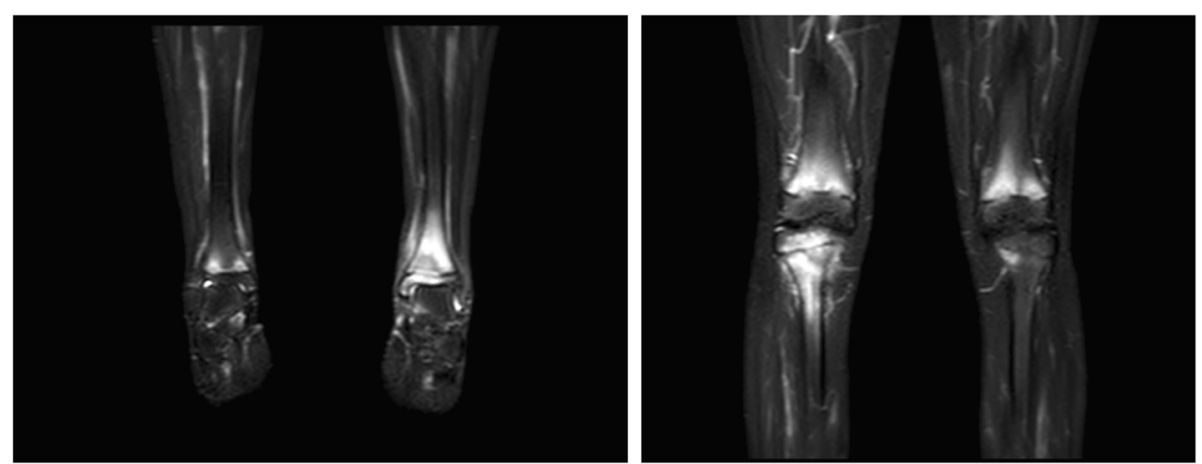

Fig. 9 STIR fa suppression image of lesions in proximal and distal metaphysis of left tibia

are the lower extremity bones. Initial symptoms including bone pain are reported in the majority of patients $[8,23,24]$.

As our patient presented multiple bone lesions, had a biopsy indicative of chronic inflammation and a negative blood culture, the diagnosis of CRMO was confirmed.

Treatment guidelines for CRMO are still under discussion and include watchful waiting for spontaneous remission, although the therapy of first choice consist of nonsteroidal anti-inflammatory drugs [28]. Glucocorticosteroids, bisphosphonates including pamidronate, sulfasalazine, anti-tumor necrosis factor $\alpha$ (TNF $\alpha)$ drugs have been used with good results (Table 1) [25-27]. Antibiotic treatment is considered ineffective [2, 6]. In our case, the patient underwent pharmacological therapy with glucocorticosteroids, sulfasalazine and pamidronate and responded well to introduced treatment. After two courses of bisphosphonates therapy - went to remission, no pain was reported, inflammation markers decreased.

\section{Conclusion}

Differential diagnosis of CRMO is challenging and it is based on exclusions. Since it might be misdiagnosed or mistreated, bone biopsy should be considered in patients reporting bone pain who are unresponsive to treatment.

Table 1 Treatment protocol for CRMO

First line treatment - NSAIDs:
Ibuprofen $30-40 \mathrm{mg} / \mathrm{kg} /$ day in $3-4$ divided doses for $1-3$ months
Naproxen $10-15 \mathrm{mg} / \mathrm{kg} /$ day in 2 divided doses for $1-3$ months
Second line treatment - Corticosteroids:
Prednisolone $1-2 \mathrm{mg} / \mathrm{kg} /$ day in 1 dose for $5-10$ days up to clinical
improvement, NSAIDs as the continuation of the therapy;
In severe cases prednisolone treatment may be prolonged up to 4-6
weeks
Third line treatment - DMARDs
Sulfasalazine
Methotrexate
Bisphosphonates (Pamidronate)
TNFa-inhibitors

\section{Abbreviations}

AFP: Alpha fetoprotein; CRMO: Chronic recurrent multifocal osteomyelitis; CRP: C-reactive protein; DWIPS: Diffusion-weighted whole-body imaging with background body signal suppression; EBV: Epstein-Barr Virus; ESR: Erythrocyte sedimentation rate; NMR: Nuclear magnetic resonance; NSE: Neuron-specific enolase; TNF: Tumor necrosis factor; VAS: Visual analogue scale

\section{Acknowledgements}

Not applicable.

\section{Authors' contributions}

J.SJ - Conception or design of the work, Data collection, Data analysis and interpretation, Drafting the article. E.S.- Critical revision of the article, Final approval of the version to be published. Both authors have read and approved the final version of the manuscript. All authors are ensure that this is the case report.

Funding

Not applicable.

\section{Availability of data and materials}

Available in the Department of Pediatric Cardiology and Rhematology, Medical Univrsity of Lodz, Poland.

Ethics approval and consent to participate Not applicable.

\section{Consent for publication}

Written, was obtained by the patients legal guardian.

\section{Competing interests}

The authors certify that they have NO affiliations with or involvement in any organization or entity with any financial interest (such as honoraria; educational grants; participation in speakers' bureaus; membership, employment, consultancies, stock ownership, or other equity interest; and expert testimony or patent-licensing arrangements), or non-financial interest (such as personal or professional relationships, affiliations, knowledge or beliefs) in the subject matter or materials discussed in this manuscript.

Received: 1 December 2018 Accepted: 22 August 2019

Published online: 31 August 2019

\section{References}

1. Fergusson PJ, Sandu M. Current understanding of the pathogenesis and Management of Chronic Recurrent Multifocal Osteomyelitis. Curr Rheumatol Rep. 2012;14:130-41.

2. Hedrich CM, Hofmann SR, Pablik J, et al. Autoinflammatory bone disorders with special focus on chronic recurrent multifocal osteomyelitis (CRMO). Pediatr Rheumatol. 2013;11:47.

3. Acikgoz G, Averill LW. Chronic recurrent multifocal osteomyelitis: typical patterns of bone involvement in whole-body bone scintigraphy. Nucl Med Commun. 2014;35:797-807. 
4. Hofmann SR, Schnabel A, Rösen-Wolff A, et al. Chronic nonbacterial osteomyelitis: pathophysiological concepts and current treatment strategies. J Rheumatol. 2016;43:1956-64.

5. Hofmann SR, Kapplusch F, Girschick HJ, et al. Chronic recurrent multifocal osteomyelitis (CRMO): presentation, pathogenesis, and treatment. Curr Osteoporos Rep. 2017;15:542-54.

6. Girschick HJ, Zimmer C, Klaus G, et al. Chronic recurrent multifocal osteomyelitis: what is it and how should it be treated? Nat Clin Pract Rheumatol. 2007:3:733-8.

7. Oligbu G, Jacobs B, Khan T. The Dilemma of Chronic Recurrent Multifocal Osteomyelitis. Reumatol Clin. 2019;18:30235-3.

8. Roderick MR, Shah R, Rogers V, et al. Chronic recurrent multifocal osteomyelitis (CRMO) - advancing the diagnosis. Pediatr Rheumatol Online J. 2016;14:47.

9. Manson D, Wilmot DM, King S, et al. Physeal involvement in chronic recurrent multifocal osteomyelitis. Pediatr Radiol. 1989:20(1-2):76-9.

10. Beretta-Piccoli BC, Sauvain MJ, Gal I, et al. Synovitis, acne, pustulosis, hyperostosis, osteitis (SAPHO) syndrome in childhood: a report of ten cases and review of the literature. Eur J Pediatr. 2000;159:594-601.

11. Aygun D, Barut K, Camcioglu Y, et al. Chronic recurrent multifocal osteomyelitis:a rare skeletal disorder. BMJ Case Rep. 2015;2015(10) bcr2015210061.

12. Alshammari A, Usmani S, Elgazzar AH, et al. Chronic recurrent multifocal osteomyelitis in children: a multidisciplinary approach is needed to establish a diagnosis. World J Nucl Med. 2013;12:120-3.

13. Jurik AG. Chronic recurrent multifocal osteomyelitis. Semin Musculoskelet Radiol. 2004:8:243-53. https://doi.org/10.1055/s-2004-835364.

14. Khanna G, Sato TS, Ferguson P. Imaging of chronic recurrent multifocal osteomyelitis. Radiographics. 2009;29:1159-77.

15. Buck FM, Treumann FC, Winiker $\mathrm{H}$, et al. Chronic recurrent multifocal osteomyelitis (CRMO) with symmetric involvement of both femora: X-ray, bone scintigram, and MR imaging findings in one case. J Magn Reson Imaging. 2007;26:422-6.

16. Catalano-Pons C, Comte A, Wipff J, et al. Clinical outcome in children with chronic recurrent multifocal osteomyelitis. Rheumatology (Oxford). 2008;47: 1397-9. https://doi.org/10.1093/rheumatology/ken249.

17. Borzutzky A, Stern S, Reiff A, et al. Pediatric chronic nonbacterial osteomyelitis. Pediatrics. 2012:130(5):e1190-7.

18. Costa-Reis P, Sullivan KE. Chronic recurrent multifocal osteomyelitis. J Clin Immunol. 2013;33(6):1043-56.

19. Padwa BL, Dentino K, Robson CD, et al. Pediatric chronic nonbacterial osteomyelitis of the jaw: clinical, radiographic, and histopathologic features. J Oral Maxillofac Surg. 2016;74(12):2393-402.

20. Gonçalves BPA, Lima MG, Nogueira CD, et al. Evolution of chronic recurrent multifocal osteomyelitis in a child shown by MRI. Radiol Case Rep. 2018;14(1):58-62

21. Fritz J, Tzaribatchev N, Claussen CD, et al. Chronic recurrent multifocal osteomyelitis: comparison of whole-body MR imaging with radiography and correlation with clinical and laboratory data. Radiology. 2009;252(3):842-51.

22. Handrick W, Hormann D, Voppmann A. Chronic recurrent multifocal osteomyelitis—report of eight patients. Pediatr Surg Int. 1998;14(3):195, 198.

23. Iyer RS, Thapa MM, Chew FS. Chronic recurrent multifocal osteomyelitis: review. Am J Roentgenol. 2011;196:87-91.

24. Yamashita K, Calderaro C, Labianca L, et al. Chronic recurrent multifocal osteomyelitis (CRMO) involving spine: a case report and literature review. J Orthop Sci. 2018. https://doi.org/10.1016/j.jos.2018.06.015.

25. Tronconi E, Miniaci A, Baldazzi M, et al. Biologic treatment for chronic recurrent multifocal osteomyelitis: report of four cases and review of the literature. Rheumatol Int. 2018 Jan;38(1):153-60. https://doi.org/10.1007/s00296-017-3877-0.

26. Miettunen PM, Wei $X$, Kaura D, et al. Dramatic pain relief and resolution of bone inflammation following pamidronate in 9 pediatric patients with persistent chronic recurrent multifocal osteomyelitis (CRMO). Pediatr Rheumatol Online J. 2009:7(2). https://doi.org/10.1186/1546-0096-7-2.

27. Taddio A, Zennaro F, Pastore S, et al. An update on the pathogenesis and treatment of chronic recurrent multifocal osteomyelitis in children. Paediatr Drugs. 2017 Jun;19(3):165-72.

28. Beck C, Morbach H, Beer M, et al. Chronic nonbacterial osteomyelitis in childhood: prospective follow-up during the first year of anti-inflammatory treatment. Arthritis Res Ther. 2010;12(2):R74.

\section{Publisher's Note}

Springer Nature remains neutral with regard to jurisdictional claims in published maps and institutional affiliations.

Ready to submit your research? Choose BMC and benefit from:

- fast, convenient online submission

- thorough peer review by experienced researchers in your field

- rapid publication on acceptance

- support for research data, including large and complex data types

- gold Open Access which fosters wider collaboration and increased citations

- maximum visibility for your research: over $100 \mathrm{M}$ website views per year

At $\mathrm{BMC}$, research is always in progress.

Learn more biomedcentral.com/submissions 